\title{
A Broadband Multilevel Fast Multipole Algorithm with Incomplete-Leaf Tree Structures for Multiscale Electromagnetic Problems
}

\author{
Manouchehr Takrimi $^{1}$, Özgür Ergül ${ }^{2}$, Vakur B. Ertürk ${ }^{1}$ \\ ${ }^{1}$ Department of Electrical and Electronics Engineering, Bilkent University, Ankara, TR-06800, Turkey \\ ${ }^{2}$ Department of Electrical and Electronics Engineering, Middle East Technical University, Ankara, TR-06800, Turkey
}

\begin{abstract}
An efficient, broadband, and accurate multilevel fast multipole algorithm (MLFMA) is proposed to solve a wide range of multiscale electromagnetic problems with orders of magnitude differences in the mesh sizes. Given a maximum RWG population threshold, only overcrowded boxes are recursively bisected into smaller ones, which leads to novel incomplete-leaf tree structures. Simulations reveal that, for surface discretizations possessing highly overmeshed local regions, the proposed method presents a more efficient and/or accurate results than the conventional MLFMA. The key feature of such a population-based clustering scenario is that the error is controllable, and hence, regardless of the number of levels, the efficiency can be optimized based on the population threshold. Numerical examples are provided to demonstrate the superior efficiency and accuracy of the proposed algorithm in comparison to the conventional MLFMA.
\end{abstract}

Index Terms-Broadband solvers, multilevel fast multipole algorithm, incomplete leaf, multiscale problems.

\section{INTRODUCTION}

The multilevel fast multipole algorithm (MLFMA) [1], its advantages [2], as well as its limitations are well known in the literature [3]. In this paper, we study one of the weaknesses of the conventional MLFMA and propose a novel broadband incomplete-leaf (IL) MLFMA for multiscale electromagnetic problems by introducing a novel concept of IL tree structures, where only overcrowded boxes are bisected into the smaller ones. For problems with multiscale features, in addition to using variable mesh sizes, variable box sizes are incorporated so that both $\mathcal{O}\left(N^{2}\right)$ complexity coming from the near-field calculations of overcrowded boxes and the computational errors arising from the protrusion of basis/testing functions are eliminated. In comparison to the conventional MLFMA, the efficiency and accuracy of the proposed IL-MLFMA are better when the multiscale factor, defined as the ratio of the largest edge length to the smallest one over the entire meshed surface, increases. An approximate method for the diagonalization of the Green's function proposed in [4], which is simple and stable at arbitrary low-frequencies, is implemented to overcome the well-known low-frequency problem of MLFMA. The IL tree structures automatically reduce to the traditional ones and the proposed IL-MLFMA reduces into the conventional MLFMA, if desired, for uniform meshes. Consequently, the proposed IL-MLFMA can also be combined with the available domain-decomposition algorithms, which are usually employed together with the conventional MLFMA to attack multiscale electromagnetic problems with very large multiscale factors.

\section{Variable Boxes for Multiscale Meshes}

We consider electromagnetic problems discretized with the Rao-Wilton-Glisson (RWG) functions [5]. In the proposed method, we modify the whole clustering strategy of the conventional MLFMA, so there is no need to divide all the boxes across a given level to create the next level. Only those boxes with the RWG populations exceeding some predetermined threshold (that may be a function of the corresponding level) are split into smaller boxes. This is achieved by letting the program start from the second level (MoM level), and continue the clustering process as far as it takes such that none of the leaf boxes are overpopulated. On the other hand, the novel IL tree structure can be reduced to a traditional one by simply assigning unity population threshold for all higher levels except the desired last level, and hence, the conventional MLFMA is recovered. Note that, because the boxes are variable sized, large boxes usually contain large RWG functions, thereby minimizing possible protrusions of the functions from the corresponding boxes.

\section{New Incomplete-Leaf Tree Structure}

To determine how deep we need to continue the clustering process, one needs to recursively slice the object in three dimensions and record the population statistics across all the levels for two important reasons: (a) To decide whether a box is an overcrowded one or not. (b) To have an educated guess about the proper value of the population threshold specific for that level. In the new IL tree structure, for a predetermined threshold, which may be fixed or a function of level, three different types of boxes are defined. The first one is an overcrowded box (OCB) that contains more (or equal) number of RWG functions than the threshold. The second one is a truncated box (TB) that has a less number of RWG functions than the threshold. A TB may exist at any level and no other boxes branches from a TB. Thus, TBs are actually new leaves but with distributed positions across different levels that makes some or many of the branches incomplete, leading us to the novel IL tree structure. Finally, a third one is a pruned box 


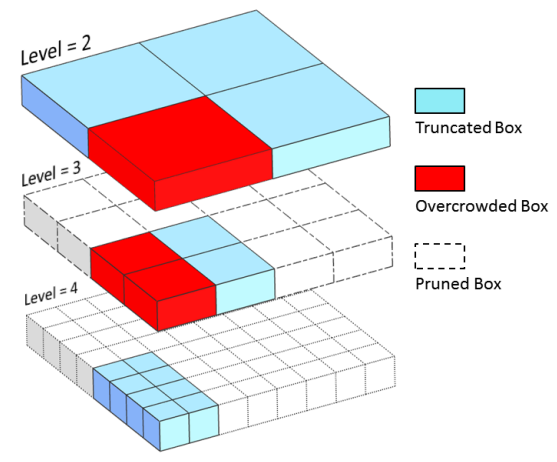

Fig. 1. A typical IL tree structure for a thin object (as a 2D example, heights are not in scale). TBs and OCBs are shown in light gray (cyan) and dark gray (red), respectively. White boxes are considered as pruned boxes.

(PB), which is a virtual box that was supposed to be one of the extensions for a TB if the tree structure was full.

Fig. 1 illustrates a typical four-level IL tree structure for a thin object (without taking into account the correct height of the boxes), where OCBs, TBs, and PBs can clearly be seen. Since an IL tree structure is different than a traditional one used by the conventional MLFMA, near-box and far-box concepts are required to be redefined. In addition, some ground rules must be constructed to determine near and far boxes from the accuracy and efficiency point of views. These are detailed in [6].

\section{NumERICAL RESULTS}

A perfect electric conductor (PEC) sphere of radius $R=$ $5 \mathrm{~cm}(0.05 \lambda)$ at $f=300 \mathrm{MHz}$ with a highly nonuniform mesh is used to demonstrate the accuracy of IL-MLFMA. Lowfrequency issue is treated exactly the same way as discussed in [4]. The edge lengths for the triangles vary from $0.03 \mathrm{~mm}$ $(\lambda / 33,300)$ at the front up to $7 \mathrm{~mm}(\lambda / 142)$ at the back of the sphere, resulting a nonuniform mesh consisting of 210,864

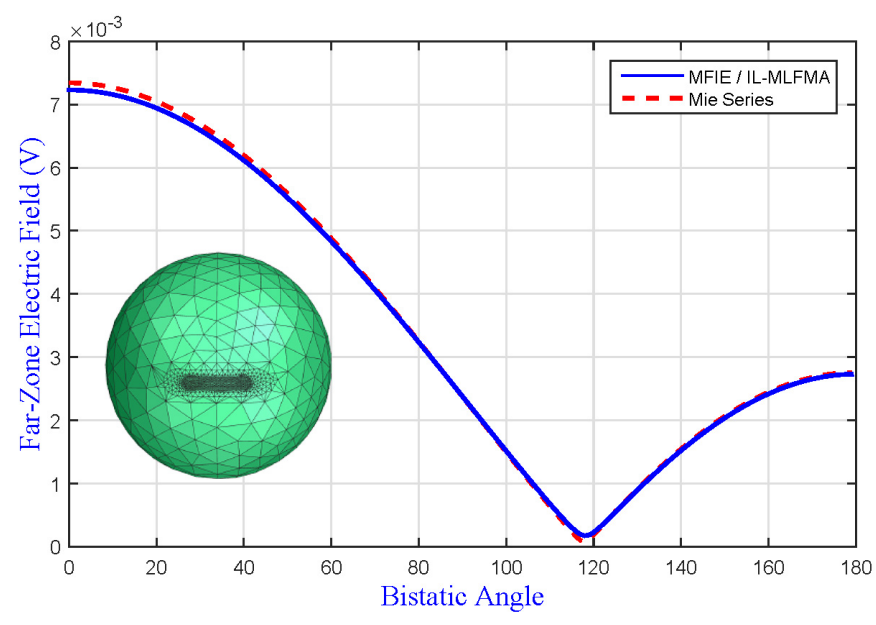

Fig. 2. Far-zone electric field scattered from a PEC sphere of radius $R=5 \mathrm{~cm}$ illuminated by a unit plane wave at $300 \mathrm{MHz}$. The sphere is nonuniformly discretized as shown in the left corner with a multiscale factor of 230 , leading to 210,864 RWG functions.
RWG functions. The magnetic-field integral equation (MFIE) formulation is used due to its stability at low frequencies. The IL tree structure consists of 10 levels with a population threshold of 100. Fig. 2 depicts the far-zone electric field as a function of the bistatic angle, along with the Mieseries solution, where $0^{\circ}$ and $180^{\circ}$ correspond to the forwardscattering and backscattering directions, respectively. MATLAB programming environment under Windows is utilized for simulation. Total run-time is about 49,180 seconds consisting of 170 seconds for tree construction, 28,180 seconds for nearfield computations, and 20,350 seconds for 52 iterations as the solution time (using GMRES method). It is seen that there is a very good agreement between Mie-series results and the values obtained with IL-MLFMA. Small amount of discrepancy is originated from the approximate method in the low-frequency regime and it is not related to the proposed IL structure.

\section{CONCLUSiON}

An efficient and versatile broadband MLFMA, referred to as IL-MLFMA, is presented to solve multiscale electromagnetic problems involving PEC objects. The concept of IL tree structures is introduced, where only the overcrowded boxes are divided into smaller ones for a given population threshold, leading to a nonuniform clustering. Thus, protrusion of the RWG functions from the boxes is minimized which improves the accuracy. Moreover, the total number of interacting boxes is reduced that improves the efficiency. The error of the proposed structure is almost independent of the levels. Consequently, for the geometries that can be discretized with nonuniform meshes with a large multiscale factor, IL-MLFMA is always more efficient than the conventional one for the same accuracy, and it is more accurate if the efficiency is comparable. Furthermore, IL-MLFMA recovers the conventional MLFMA for uniform meshes, if desired. As a result, all other computational methods that can be combined with the conventional MLFMA can also be used in conjunction with the proposed IL-MLFMA.

\section{REFERENCES}

[1] W. C. Chew, E. Michielssen, J. Song, and J. Jin, Fast and Efficient Algorithms in Computational Electromagnetics. Boston-London: Artech House Publisher, 2001.

[2] W. C. Chew and L. Jiang, "Overview of large-scale computing: the past, the present, and the future," Proc. IEEE, vol. 101, no. 2, pp. 227-241, Feb. 2013.

[3] Ö. Ergül and L. Gürel, The Multilevel Fast Multipole Algorithm (MLFMA) for Solving Large-Scale Computational Electromagnetics Problems. Wiley-IEEE Press, 2014.

[4] Ö. Ergül and B. Karaosmanoğlu, "Approximate stable diagonalization of the Green's function for low frequencies," IEEE Antennas Wirel. Propag. Lett., vol. 13, no. 2, pp. 1054-1056, 2014.

[5] S. Rao, D. Wilton, and A. Glisson, "Electromagnetic scattering by surfaces of arbitrary shape," IEEE Trans. Antennas Propag., vol. 30, no. 3, pp. 409-418, May 1982.

[6] M. Takrimi, Ö. Ergül, and V. B. Ertürk, "A novel broadband multilevel fast multipole algorithm with incomplete-leaf tree structures for multiscale electromagnetic problems," unpublished. 